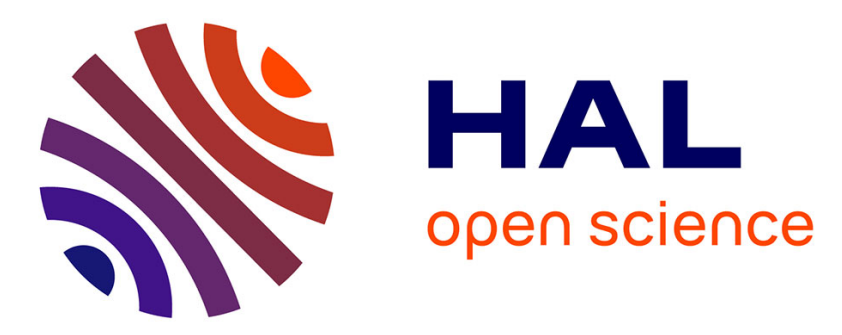

\title{
Effect of coupling with internal and external fluids on the mechanical behavior of aerostats
}

\author{
Jean-Sébastien Schotté, Robin Le Mestre, Olivier Doaré
}

\section{To cite this version:}

Jean-Sébastien Schotté, Robin Le Mestre, Olivier Doaré. Effect of coupling with internal and external fluids on the mechanical behavior of aerostats. SEMC 2019, Sep 2019, CAPE TOWN, South Africa. hal-02420661

\section{HAL Id: hal-02420661 \\ https://hal.science/hal-02420661}

Submitted on 20 Dec 2019

HAL is a multi-disciplinary open access archive for the deposit and dissemination of scientific research documents, whether they are published or not. The documents may come from teaching and research institutions in France or abroad, or from public or private research centers.
L'archive ouverte pluridisciplinaire HAL, est destinée au dépôt et à la diffusion de documents scientifiques de niveau recherche, publiés ou non, émanant des établissements d'enseignement et de recherche français ou étrangers, des laboratoires publics ou privés. 


\title{
Effect of coupling with internal and external fluids on the mechanical behavior of aerostats
}

\author{
J.-S. Schotté \\ Aerodynamics, Aeroelasticity and Acoustics Department \\ ONERA, Châtillon, France
}

R. Le Mestre \& O. Doaré

Unité de Mécanique

ENSTA ParisTech, Palaiseau, France

\begin{abstract}
In the context of the simulation of aerostats in flight, we are interested here in the coupling between a deformable structure, the fluid contained inside and the fluid flow outside. To study the dynamic stability of such systems, the fluid-structure coupled equations are linearized around an equilibrium position and, by assuming that the fluid flow perturbations are potential, the loads exerted by the fluids on the moving structure can be decomposed in terms proportional, respectively, to the displacement, velocity and acceleration fields of the structure, representing what are generally called the added stiffness, damping and mass effects of the fluid on the structure. In this work, a focus is made on the added mass because, for such lightweight structures, its effect is of prime importance. A Boundary Element Method (BEM) is proposed to compute the fluid added mass operators, for external and internal fluids, and for any structure deformation field. Numerical and experimental validations are conducted on an axisymmetric ellipsoid mockup immerged in water and subject to rigid motions. Variations of the imposed movement amplitude and velocity have also helped to evaluate the validity domain of this model.
\end{abstract}

\section{INTRODUCTION}

In the context of the simulation of aerostats in flight, we are interested here in the coupling between the elastic deformations of the airshsip hull and the pressure forces, due to helium inside and to air flow outside. Because flow speed is generally much smaller for aerostat than for aerodyn, it is generally consider that the main effect of these fluids on the structure is an added mass effect that can be estimated with a model of potential fluid at rest (Hembree \& Slegers 2011). The objectives of this paper are: to clarify the assumptions needed to justify this approach, to remind briefly how to compute the added mass operator for an external fluid by the Boundary Element Method (and to propose an extension to an internal fluid), to experimentally evaluate the validity domain of this model, and finally to express the other added stiffness and damping terms that will be studied in future works.

\section{VIBRATIONS OF A STRUCTURE IN A PERMANENT FLUID FLOW}

In this study, the fluid velocity is supposed to be significantly smaller than the sound speed (Mach number $\ll 1$.): the fluid is then considered incompressible, which means that fluid density $\rho$ is a constant

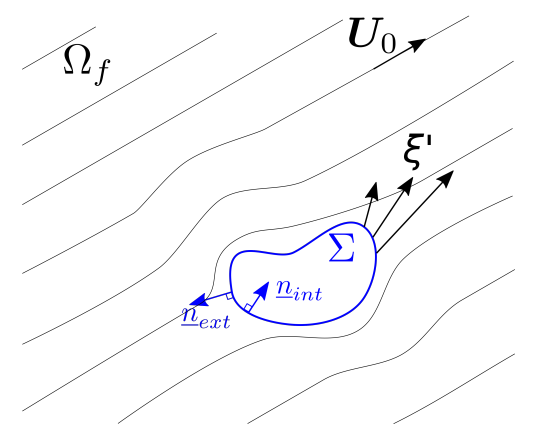

Figure 1: Structure in vibrations in a permanent fluid flow

over time and space, and consequently, the continuity equation provides the relation $\boldsymbol{\nabla} \cdot \boldsymbol{u}=0$, where $\boldsymbol{u}$ is the fluid velocity. The incompressible Navier-Stokes equation is then written

$\rho \frac{\partial \boldsymbol{u}}{\partial t}+\rho(\boldsymbol{u} . \nabla) \boldsymbol{u}=-\nabla p-\rho g \boldsymbol{e}_{z}+\mu \Delta \boldsymbol{u}$

where $p$ is the pressure field and $\mu$ the fluid dynamic viscosity. Let us remark that coupling with thermic phenomena is disregarded here.

The permanent flow around the structure at rest is supposed to be known ${ }^{1}$ and characterized by a velocity field $\boldsymbol{u}_{0}$ and a pressure field $p_{0}$ solutions of the

\footnotetext{
${ }^{1}$ It can be the result of a stationary Computational Fluid Dynamics simulation for example.
} 
stationary equations

$$
\begin{aligned}
\rho\left(\boldsymbol{u}_{0} . \boldsymbol{\nabla}\right) \boldsymbol{u}_{0} & =-\boldsymbol{\nabla} p_{0}-\rho g \boldsymbol{e}_{z}+\mu \Delta \boldsymbol{u}_{0} \\
\boldsymbol{\nabla} \cdot \boldsymbol{u}_{0} & =0
\end{aligned}
$$

The structure position in this equilibrium state is supposed to be known as well and taken as the reference to describe the structure vibrations $\left(\boldsymbol{\xi}_{0}=0\right)$. Some prestresses, due to static pressures acting on the walls, exist in the structure and are denoted as $\boldsymbol{\sigma}_{0}$.

We are interested here in small perturbations of the fluid due to small amplitude vibrations of the structure $\boldsymbol{\xi}^{\prime}$. The associated pressure and velocity fluctuations in the fluid flow will be denoted respectively as $p^{\prime}$ and $\boldsymbol{u}^{\prime}$ such that: $\boldsymbol{u}=\boldsymbol{u}_{0}+\boldsymbol{u}^{\prime}$ and $p=p_{0}+p^{\prime}$. By introducing this decomposition and using the equations of the stationary solution (2), we get the equations of the perturbated flow:

$$
\begin{gathered}
\rho\left[\frac{\partial \boldsymbol{u}}{\partial t}+\left(\boldsymbol{u}_{0} \cdot \nabla\right) \boldsymbol{u}^{\prime}+\left(\boldsymbol{u}^{\prime} . \nabla\right) \boldsymbol{u}_{0}+\left(\boldsymbol{u}^{\prime} . \nabla\right) \boldsymbol{u}^{\prime}\right]=\cdots \\
-\nabla p^{\prime}+\mu \Delta \boldsymbol{u}^{\prime} \quad \text { in } \Omega_{f 0}
\end{gathered}
$$

$\boldsymbol{\nabla} \cdot \boldsymbol{u}^{\prime}=0 \quad$ in $\Omega_{f 0}$

We suppose that structure vibrations are characterized by a pulsation $\Omega_{0}$ and an amplitude $\Xi_{0}$ and we introduce nondimensionalized variables defined by $\boldsymbol{\xi}^{\prime}=\Xi_{0} \overline{\boldsymbol{\xi}}^{\prime}, \boldsymbol{u}^{\prime}=\Xi_{0} \Omega_{0} \overline{\boldsymbol{u}}^{\prime}, \boldsymbol{u}_{0}=U_{0} \overline{\boldsymbol{u}}_{0}, p^{\prime}=\rho L \Xi_{0} \Omega_{0}^{2} \bar{p}^{\prime}$, where $U_{0}$ is the upstream velocity and $L$ is a structure characteristic length. These nondimensionalized variables have been constructed such that their order of magnitude is close to 1 . Nondimensionalized time and space variables are also defined by $\bar{t}=\Omega_{0} t, \boldsymbol{x}=L \overline{\boldsymbol{x}}$. The nondimensionalized equations of the perturbated flow are then given by

$$
\begin{aligned}
\frac{\partial \overline{\boldsymbol{u}}^{\prime}}{\partial t}+ & U_{R}\left[\left(\overline{\boldsymbol{u}}^{\prime} \cdot \boldsymbol{\nabla}\right) \overline{\boldsymbol{u}}_{0}+\left(\overline{\boldsymbol{u}}_{0} \cdot \boldsymbol{\nabla}\right) \overline{\boldsymbol{u}}^{\prime}\right]+\mathcal{D}\left(\overline{\boldsymbol{u}}^{\prime} \cdot \boldsymbol{\nabla}\right) \overline{\boldsymbol{u}}^{\prime}=\cdots \\
& -\boldsymbol{\nabla} \bar{p}^{\prime}+\frac{1}{S t} \Delta \overline{\boldsymbol{u}}^{\prime}
\end{aligned}
$$

$$
\nabla \cdot \overline{\boldsymbol{u}}^{\prime}=0
$$

where $S t$ is the Stokes number, $\mathcal{D}$ the displacement number and $U_{R}$ the reduced velocity, respectively defined by

$S t=\frac{\rho L^{2} \Omega_{0}}{\mu}, \quad \mathcal{D}=\frac{\Xi_{0}}{L}, \quad U_{R}=\frac{U_{0}}{L \Omega_{0}}$

In the following, we will suppose ${ }^{2}$ that viscosity forces in the fluid are negligible with respect to inertia forces, i.e. $S t \gg 1$, and that the structure displacement amplitudes are small with respect to the structure size,

\footnotetext{
${ }^{2}$ These assumptions will be discussed in section 4 .
}

i.e. $\mathcal{D} \ll 1$. After simplification of relation (4a) and switch back to dimensionalized variables, we finally obtain the expression of the gradient of the pressure fluctuation in the perturbated flow:

$\boldsymbol{\nabla} p^{\prime}=-\rho\left[\frac{\partial \boldsymbol{u}^{\prime}}{\partial t}+\left(\boldsymbol{u}_{0} \cdot \boldsymbol{\nabla}\right) \boldsymbol{u}^{\prime}+\left(\boldsymbol{u}^{\prime} \cdot \boldsymbol{\nabla}\right) \boldsymbol{u}_{0}\right]$

where $\boldsymbol{u}^{\prime}$ is the solution of equation (3b) completed by boundary conditions.

Far from the structure, we consider that the flow perturbations vanish, what can be transcribed by the following far-field conditions (deduced from Sommerfeld's radiation conditions applied to an incompressible fluid):

$p^{\prime}=\mathcal{O}\left(\frac{1}{R}\right)$ and $\left|\boldsymbol{u}^{\prime}\right|=\mathcal{O}\left(\frac{1}{R^{2}}\right)$ when $R \rightarrow \infty$

where $R$ is the distance with respect to the structure.

Because of the assumption that viscosity forces in the fluid are negligible $(S t \gg 1)$, the continuity condition at the interface between the vibrating structure and the fluid flow will be imposed only on the normal component of the velocity:

$\boldsymbol{u} . \boldsymbol{n}=\frac{\partial \boldsymbol{\xi}^{\prime}}{\partial t} \cdot \boldsymbol{n} \quad$ on $\Sigma$

By introducing the decomposition of $\boldsymbol{u}$ and the nondimensionalization, an equivalent nondimensionalized expression is obtained

$$
\left(\overline{\boldsymbol{u}}_{0}+\frac{\mathcal{D}}{U_{R}} \overline{\boldsymbol{u}}^{\prime}\right) \cdot \boldsymbol{n}=\frac{\mathcal{D}}{U_{R}} \frac{\partial \overline{\boldsymbol{\xi}}^{\prime}}{\partial \bar{t}} \cdot \boldsymbol{n} \quad \text { on } \bar{\Sigma}
$$

This relation is written on the instantaneous position of the interface, however, because we consider small displacements of the structure, the initial (equilibrium) position $\Sigma_{0}$ of this interface is the reference and then it is necessary to transfer that relation on this reference position. For this purpose, we introduce the instantaneous position $\boldsymbol{x}$, and the initial one $\boldsymbol{x}_{0}$, of the interface, which, in nondimensionalized version, are linked by $\overline{\boldsymbol{x}}=\overline{\boldsymbol{x}}_{0}+\mathcal{D} \overline{\boldsymbol{\xi}}^{\prime}$. Since $\mathcal{D} \ll 1$, we can use a first order Taylor expansion of terms in (9):

$$
\begin{aligned}
& \overline{\boldsymbol{u}}_{0}(\overline{\boldsymbol{x}})=\overline{\boldsymbol{u}}_{0}\left(\overline{\boldsymbol{x}}_{0}\right)+\mathcal{D}\left(\overline{\boldsymbol{\xi}}^{\prime} \cdot \nabla\right) \overline{\boldsymbol{u}}_{0}\left(\overline{\boldsymbol{x}}_{0}\right)+o(\mathcal{D}) \\
& \overline{\boldsymbol{u}}^{\prime}(\overline{\boldsymbol{x}})=\overline{\boldsymbol{u}}^{\prime}\left(\overline{\boldsymbol{x}}_{0}\right)+\mathcal{D}\left(\overline{\boldsymbol{\xi}}^{\prime} \cdot \boldsymbol{\nabla}\right) \overline{\boldsymbol{u}}^{\prime}\left(\overline{\boldsymbol{x}}_{0}\right)+o(\mathcal{D}) \\
& \boldsymbol{n} \mathrm{d} \bar{\Sigma}=\boldsymbol{n}_{0} \mathrm{~d} \bar{\Sigma}_{0}+\mathcal{D} \boldsymbol{\tau}\left(\overline{\boldsymbol{\xi}}^{\prime}\right) \mathrm{d} \bar{\Sigma}_{0}+o(\mathcal{D}) \\
& \frac{\partial \overline{\boldsymbol{\xi}}^{\prime}}{\partial t}(\overline{\boldsymbol{x}})=\frac{\partial \overline{\boldsymbol{\xi}}^{\prime}}{\partial t}\left(\overline{\boldsymbol{x}}_{0}\right)+\mathcal{D}\left(\overline{\boldsymbol{\xi}}^{\prime} \cdot \nabla\right) \frac{\partial \boldsymbol{\xi}^{\prime}}{\partial t}\left(\overline{\boldsymbol{x}}_{0}\right)+o(\mathcal{D})
\end{aligned}
$$

where vector $\tau$ is defined in (Morand \& Ohayon $1995)$ as the vector of normal rotation whose expression is given by

$$
\boldsymbol{\tau}\left(\overline{\boldsymbol{\xi}}^{\prime}\right)=\left(\boldsymbol{\nabla} \cdot \overline{\boldsymbol{\xi}}^{\prime}\right) \boldsymbol{n}_{0}-{ }^{t} \underline{\underline{\nabla} \overline{\boldsymbol{\xi}^{\prime}}} \boldsymbol{n}_{0}
$$


By introducing these expansions in (9) and keeping only the terms of the first order in $\mathcal{D}$, we obtain

$$
\begin{gathered}
\overline{\boldsymbol{u}}_{0}\left(\overline{\boldsymbol{x}}_{0}\right) \cdot \boldsymbol{n}_{0}+\mathcal{D} \boldsymbol{n}_{0} \cdot\left(\overline{\boldsymbol{\xi}}^{\prime} \cdot \nabla\right) \overline{\boldsymbol{u}}_{0}\left(\overline{\boldsymbol{x}}_{0}\right)+\mathcal{D} \overline{\boldsymbol{u}}_{0}\left(\overline{\boldsymbol{x}}_{0}\right) \cdot \boldsymbol{\tau}\left(\overline{\boldsymbol{\xi}}^{\prime}\right) \\
+\frac{\mathcal{D}}{U_{R}} \overline{\boldsymbol{u}}^{\prime}\left(\overline{\boldsymbol{x}}_{0}\right) \cdot \boldsymbol{n}_{0}=\frac{\mathcal{D}}{U_{R}} \frac{\partial \boldsymbol{\xi}^{\prime}}{\partial t}\left(\overline{\boldsymbol{x}}_{0}\right) \cdot \boldsymbol{n}_{0}+o(\mathcal{D})
\end{gathered}
$$

As $\overline{\boldsymbol{u}}_{0} \cdot \boldsymbol{n}_{0}=0$ (since the permanent flow cannot penetrate or separate from the structure), the boundary condition for the perturbated flow at the interface with the moving structure is finally given on $\Sigma_{0}$ (in dimensionalized variables) by:

$$
\boldsymbol{u}^{\prime} \cdot \boldsymbol{n}_{0}=\frac{\partial \boldsymbol{\xi}^{\prime}}{\partial t} \cdot \boldsymbol{n}_{0}-\boldsymbol{n}_{0} \cdot\left(\boldsymbol{\xi}^{\prime} \cdot \nabla\right) \boldsymbol{u}_{0}-\boldsymbol{u}_{0} \cdot \boldsymbol{\tau}\left(\boldsymbol{\xi}^{\prime}\right)
$$

The pressure and velocity perturbations, $p^{\prime}$ and $\boldsymbol{u}^{\prime}$, are finally the solutions of the system of linear equations (3b), (6), (7) and (13). In the following, we will do the hypothesis that the perturbated flow is irrotational, i.e. $\boldsymbol{u}^{\prime}=\nabla \psi^{\prime}$. In the particular case of a potential permanent flow (i.e. $\boldsymbol{u}_{0}=\nabla \psi_{0}$ ), the irrotationality of the perturbation $\boldsymbol{u}^{\prime}$ is ensured by Lagrange's Theorem ${ }^{3}$ (Barenblatt 2014). Irrotationality of the perturbated flow is obviously not true in areas where flow separation occurs (with vortex shedding) and this assumption certainly limits this model to streamlined body with a reasonable angle of attack with respect to the flow.

Furthermore, since boundary condition (13) shows that the structure imposes its normal displacement and velocity to the fluid at the interface, the linearity of those equations can be used to decompose the fluid response as follows:

$$
\boldsymbol{u}^{\prime}=\nabla \psi^{\prime}=\nabla \psi_{1}\left(\dot{\boldsymbol{\xi}}^{\prime}\right)+\nabla \psi_{2}\left(\boldsymbol{\xi}^{\prime}\right) .
$$

where $\psi_{1}$ and $\psi_{2}$ are the velocity potentials associated with the perturbations of the flow, respectively due to the velocity of the interface, denoted as $\dot{\xi}^{\prime}$, and to its displacement $\boldsymbol{\xi}^{\prime}$. By introducing this decomposition in (3b), (7) and (13) and separating the terms in $\dot{\xi}^{\prime}$ and $\xi^{\prime}$, we get two distinct problems associated with the following systems of equations:

$$
\begin{cases}\Delta \psi_{1}=0 & \text { in } \Omega_{f 0} \\ \boldsymbol{\nabla} \psi_{1} \cdot \boldsymbol{n}_{0}=\dot{\boldsymbol{\xi}}^{\prime} \cdot \boldsymbol{n} & \text { on } \Sigma_{0} \\ \text { ext. fluid: }\left|\boldsymbol{\nabla} \psi_{1}\right|=\mathcal{O}\left(\frac{1}{R^{2}}\right) & \text { when } R \rightarrow \infty \\ \text { ext. fluid: } \psi_{1}=\mathcal{O}\left(\frac{1}{R}\right) & \text { when } R \rightarrow \infty \\ \text { int. fluid: } \ell\left(\psi_{1}\right)=0 & \end{cases}
$$

${ }^{3}$ Lagrange's Theorem specifies that, in a conservative force field, if the flow of an homogeneous barotropic (or incompressible) perfect fluid is irrotational at a given time, it remains irrotational.

$$
\begin{cases}\Delta \psi_{2}=0 & \text { in } \Omega_{f 0} \\ \boldsymbol{\nabla} \psi_{2} \cdot \boldsymbol{n}_{0}=-\boldsymbol{n}_{0} \cdot\left(\boldsymbol{\xi}^{\prime} \cdot \boldsymbol{\nabla}\right) \boldsymbol{u}_{0}-\boldsymbol{u}_{0} \cdot \boldsymbol{\tau}\left(\boldsymbol{\xi}^{\prime}\right) \text { on } \Sigma_{0} \\ \text { ext. fluid: }\left|\boldsymbol{\nabla} \psi_{2}\right|=\mathcal{O}\left(\frac{1}{R^{2}}\right) & \text { when } R \rightarrow \infty \\ \text { ext. fluid: } \psi_{2}=\mathcal{O}\left(\frac{1}{R}\right) & \text { when } R \rightarrow \infty \\ \text { int. fluid: } \ell\left(\psi_{2}\right)=0 & \end{cases}
$$

The last two equations of each previous systems have been added to ensure the unicity of the solutions $\psi_{1}$ and $\psi_{2}$. Without these unicity conditions, those systems of equations would not allow determinating unique solutions: since $\psi_{1}$ and $\psi_{2}$ only appear through their spacial derivatives, they would be defined up to an additive constant. In the case of an external fluid, the solution unicity can be obtained by imposing a decrease of $\psi$ to 0 far from the structure, but for an internal fluid, a specific unicity condition, denoted for now as $\ell(\psi)=0$ will have to be specified later.

Now, we consider $W_{p}$ which is the virtual work of the pressure $p$ exerted on the structure for a virtual displacement $\delta \boldsymbol{\xi}$ of this one. Its expression is given by

$$
W_{p}=\int_{\Sigma}\left(p_{0}+p^{\prime}\right) \boldsymbol{n}^{f} . \delta \boldsymbol{\xi} \mathrm{d} \Sigma
$$

where $\boldsymbol{n}^{f}$ is the outwards normal to the fluid. The terms in this expression are expressed on the deformed interface $\Sigma$, and then we have to nondimensionalize it and use Taylor expansions to obtain a transposed expression (at the first order in $\mathcal{D}$ ) on the known interface position $\Sigma_{0}$. From the boundary conditions on $\psi_{1}$ and $\psi_{2}$, given in (15) and (16), we can deduce their respective order of magnitude and defined nondimensionalized variables $\psi_{1}=L \Xi_{0} \Omega_{0} \bar{\psi}_{1}$, $\psi_{2}=U_{0} \Xi_{0} \bar{\psi}_{2}, p_{0}=\rho U_{0}^{2} \bar{p}_{0}$ and $W_{p}=\rho U_{0}^{2} L^{2} \bar{W}_{p}$. By using also the previously non dimensionalized variables, we get

$$
\bar{W}_{p}=\int_{\bar{\Sigma}}\left(\bar{p}_{0}+\frac{\mathcal{D}}{U_{R}^{2}} \bar{p}^{\prime}\right) \boldsymbol{n}^{f} . \delta \boldsymbol{\xi} \mathrm{d} \bar{\Sigma}
$$

A Taylor expansion at the first order in $\mathcal{D}$ yields

$$
\begin{aligned}
\bar{p}_{0}(\overline{\boldsymbol{x}}) & =\bar{p}_{0}\left(\overline{\boldsymbol{x}}_{0}\right)+\mathcal{D} \boldsymbol{\nabla} \bar{p}_{0}\left(\overline{\boldsymbol{x}}_{0}\right) \cdot \overline{\boldsymbol{\xi}}^{\prime}+o(\mathcal{D}) \\
\bar{p}^{\prime}(\overline{\boldsymbol{x}}) & =\bar{p}^{\prime}\left(\overline{\boldsymbol{x}}_{0}\right)+\mathcal{D} \boldsymbol{\nabla} \bar{p}^{\prime}\left(\overline{\boldsymbol{x}}_{0}\right) \cdot \overline{\boldsymbol{\xi}}^{\prime}+o(\mathcal{D})
\end{aligned}
$$

By introducing these relations and (10) in (18), and discarding the terms of second order and higher in $\mathcal{D}$, the following expression is obtained

$$
\begin{aligned}
\bar{W}_{p}=\int_{\bar{\Sigma}_{0}} \bar{p}_{0}\left(\boldsymbol{n}_{0}^{f}+\mathcal{D} \boldsymbol{\tau}\left(\overline{\boldsymbol{\xi}}^{\prime}\right)\right) \cdot \delta \boldsymbol{\xi}+\cdots \\
\mathcal{D}\left(\boldsymbol{\nabla} \bar{p}_{0} \cdot \overline{\boldsymbol{\xi}}^{\prime}+\frac{1}{U_{R}^{2}} \bar{p}^{\prime}\right) \boldsymbol{n}_{0}^{f} . \delta \boldsymbol{\xi} \mathrm{d} \bar{\Sigma}_{0}
\end{aligned}
$$


If we suppose that the fluid response to the structure motions has been determined by solving the systems of equations (15) and (16) (details on the numerical resolution will be given in $\S 3$ ), the expression of the pressure fluctuation $p^{\prime}$, as a function of the structure displacements $\boldsymbol{\xi}^{\prime}$ and its time derivatives, can be obtained through a spatial integration of (6). For this purpose, the relation $\boldsymbol{\nabla}(\boldsymbol{a} . \boldsymbol{b})=(\boldsymbol{a} . \boldsymbol{\nabla}) \boldsymbol{b}+(\boldsymbol{b} . \boldsymbol{\nabla}) \boldsymbol{a}+$ $\boldsymbol{a} \wedge(\boldsymbol{\nabla} \wedge \boldsymbol{b})+\boldsymbol{b} \wedge(\nabla \wedge \boldsymbol{a})$ is used:

$\boldsymbol{\nabla}\left(\frac{-p^{\prime}}{\rho}\right)=\nabla \dot{\psi}^{\prime}+\nabla\left(\boldsymbol{u}_{0} \cdot \nabla \psi^{\prime}\right)-\nabla \psi^{\prime} \wedge\left(\nabla \wedge \boldsymbol{u}_{0}\right)$

If the permanent flow $\boldsymbol{u}_{0}$ is potential (or close to be), the last term of this expression can be neglected and we obtain Bernoulli's equation for the perturbated flow:

$p^{\prime}=-\rho\left(\dot{\psi}^{\prime}+\boldsymbol{u}_{\mathbf{0}} \cdot \boldsymbol{\nabla} \psi^{\prime}\right)+\pi(t) \quad$ in $\Omega_{f 0}$

where $\pi(t)$ is an uniform pressure in the fluid domain. In case of an external fluid, with the far-field conditions (7) and the unicity conditions on $\psi_{1}$ and $\psi_{2}$ such that $\psi=\psi_{1}+\psi_{2}=\mathcal{O}\left(\frac{1}{R}\right)$ when $R \rightarrow \infty$, the value of $\pi$ can be shown to be equal to 0 , but that is not always the case for an internal fluid.

If the permanent flow $\boldsymbol{u}_{0}$ is not potential, a correction term has to be taken into account in (21) but we can remark that this term depends on $\psi^{\prime}$ and not on its time derivatives.

Since velocity potential $\psi^{\prime}$ is decomposed in two functions $\psi_{1}$ and $\psi_{2}$ depending linearly on, respectively, the velocity $\dot{\xi}$ and the displacement $\boldsymbol{\xi}$ of the structure, see (14), its first time derivative can be directly expressed as a function of the acceleration and velocity of the structure:

$\dot{\psi}^{\prime}=\psi_{1}(\ddot{\boldsymbol{\xi}})+\psi_{2}(\dot{\boldsymbol{\xi}})$

Under the assumption of small amplitude vibrations of the system $(\mathcal{D} \ll 1)$, the variational formulation obtained from the linearized (and conservative) structure equations by the virtual work principle is given by:

$\exists \boldsymbol{\xi}^{\prime}$, such that $\forall \delta \boldsymbol{\xi}, \quad K_{S}\left(\boldsymbol{\xi}^{\prime}, \delta \boldsymbol{\xi}\right)+M_{S}\left(\ddot{\boldsymbol{\xi}}^{\prime}, \delta \boldsymbol{\xi}\right)=\cdots$

$$
W_{p}-\int_{\Sigma_{0}} p_{0} \boldsymbol{n}_{0}^{f} . \delta \boldsymbol{\xi} \mathrm{d} \Sigma_{0}+f_{\text {ext }}(\delta \boldsymbol{\xi})
$$

where $K_{S}, M_{S}$ and $f_{\text {ext }}$ are the classical stiffness ${ }^{4}$, mass and external load operators.

By introducing the dimensionalized counterpart of (20) in this expression, we identify terms in $\boldsymbol{\xi}^{\prime}$ which

\footnotetext{
${ }^{4}$ The stiffness operator contains the elastic stiffness but also the geometric stiffness related to the presence of a prestress $\boldsymbol{\sigma}_{0}$ in the structure at the equilibrium state.
}

define a new stiffness operator related to the presence of static pressure field $p_{0}$ (Morand \& Ohayon 1995):

$$
\begin{array}{r}
\forall \delta \boldsymbol{\xi}, \quad\left(K_{S}+K_{p_{0}}\right)\left(\boldsymbol{\xi}^{\prime}, \delta \boldsymbol{\xi}\right)+M_{S}\left(\ddot{\boldsymbol{\xi}}^{\prime}, \delta \boldsymbol{\xi}\right)=\cdots \\
\int_{\Sigma_{0}} p^{\prime} \boldsymbol{n}_{0}^{f} . \delta \boldsymbol{\xi} \mathrm{d} \Sigma_{0}+f_{\text {ext }}(\delta \boldsymbol{\xi})
\end{array}
$$

where $K_{p_{0}}$ contains a term proportional to the gradient of $p_{0}$ (gradient stiffness) and a term depending on the normal rotation $\tau$ (follower force stiffness):

$$
K_{p_{0}}\left(\boldsymbol{\xi}^{\prime}, \delta \boldsymbol{\xi}\right)=-\int_{\Sigma_{0}}\left(\boldsymbol{\nabla} p_{0} \cdot \boldsymbol{\xi}^{\prime}\right) \boldsymbol{n}_{0}^{f} . \delta \boldsymbol{\xi}+p_{0} \boldsymbol{\tau}\left(\boldsymbol{\xi}^{\prime}\right) . \delta \boldsymbol{\xi} \mathrm{d} \Sigma_{0}
$$

By introducing in (24) the expression (21) of $p^{\prime}$ and the decomposition (22) of $\dot{\psi}^{\prime}$, we remark that the coupling term representing the action of the fluid flow on the vibrating structure generates a supplementary stiffness operator, as well as a damping operator and an added mass operator which involve respectively the displacement $\boldsymbol{\xi}^{\prime}$, the velocity $\dot{\boldsymbol{\xi}}^{\prime}$ and the acceleration $\ddot{\xi}^{\prime}$ of the structure wall:

$$
\begin{aligned}
& \forall \delta \boldsymbol{\xi}, \quad\left(K_{S}+K_{p_{0}}+K_{u_{0}}\right)\left(\boldsymbol{\xi}^{\prime}, \delta \boldsymbol{\xi}\right)+D_{F}\left(\dot{\boldsymbol{\xi}}^{\prime}, \delta \boldsymbol{\xi}\right) \cdots \\
& +\left(M_{S}+M_{F}\right)\left(\ddot{\boldsymbol{\xi}}^{\prime}, \delta \boldsymbol{\xi}\right)=\pi \int_{\Sigma_{0}} \boldsymbol{n}_{0}^{f} . \delta \boldsymbol{\xi} \mathrm{d} \Sigma_{0}+f_{\mathrm{ext}}(\delta \boldsymbol{\xi})
\end{aligned}
$$

where the added stiffness and added damping operators are given by

$$
\begin{aligned}
& K_{u_{0}}\left(\boldsymbol{\xi}^{\prime}, \delta \boldsymbol{\xi}\right)=\int_{\Sigma_{0}} \rho u_{0} . \nabla \psi_{2}\left(\boldsymbol{\xi}^{\prime}\right)\left(\boldsymbol{n}_{0}^{f} . \delta \boldsymbol{\xi}\right) \mathrm{d} \Sigma_{0} \\
& D_{F}\left(\dot{\boldsymbol{\xi}}^{\prime}, \delta \boldsymbol{\xi}\right)=\int_{\Sigma_{0}} \rho\left(u_{0} . \nabla \psi_{1}\left(\dot{\boldsymbol{\xi}}^{\prime}\right)+\psi_{2}\left(\dot{\boldsymbol{\xi}}^{\prime}\right)\right) \boldsymbol{n}_{0}^{f} . \delta \boldsymbol{\xi} \mathrm{d} \Sigma_{0}
\end{aligned}
$$

Let us remark that these expressions suppose that the permanent fluid flow $\boldsymbol{u}_{0}$ is potential (or close to be), otherwise, a correction due to vorticity should be taken into account. However, the added mass operator $M_{F}$, whose expression is given by

$$
M_{F}\left(\ddot{\boldsymbol{\xi}}^{\prime}, \delta \boldsymbol{\xi}\right)=\int_{\Sigma_{0}} \rho \psi_{1}\left(\ddot{\boldsymbol{\xi}}^{\prime}\right) \boldsymbol{n}_{0}^{f} . \delta \boldsymbol{\xi} \mathrm{d} \Sigma_{0}
$$

depends neither on the permanent flow velocity nor on its vorticity. This important remark will allow us to compute, in the following, the added mass of fluids in contact with structures as if there were no flow (as long as the assumptions of this model are true, i.e. $\mathcal{D} \ll 1$ and $S t \gg 1$ ). 


\section{NUMERICAL COMPUTATION OF FLUID ADDED MASS}

In previous works, a Finite Element method (FEM) as been proposed to compute the added mass operator of internal fluids (Schotté \& Ohayon 2009), however, since the objective here is to construct this operator for both internal and external fluids, a Boundary Element Method (BEM) seems most suitable.

This approach is based on the integral representation of the solutions of Laplace equation. We will use this approch to solve equations (15) for any wall motion $\xi^{\prime}$ and to numerically construct the linear function $\psi_{1}\left(\dot{\boldsymbol{\xi}}^{\prime}\right)$. The integral representation method (Hackbusch 1995) states that the solution $\varphi$ of a Laplace Equation $\Delta \varphi=0$, defined on a domain $\Omega$ of the three-dimensional space, can be expressed as a function of its value and the value of its normal derivative on the boundary $\partial \Omega$, by the following relation

$\alpha_{f(\boldsymbol{y})} \varphi_{(\boldsymbol{y})}=\int_{\partial \Omega} \frac{\partial \varphi}{\partial \boldsymbol{n}_{x}^{f}} \frac{1}{|\boldsymbol{x}-\boldsymbol{y}|}+\varphi_{(\boldsymbol{x})} \frac{(\boldsymbol{x}-\boldsymbol{y}) \cdot \boldsymbol{n}_{x}^{f}}{|\boldsymbol{x}-\boldsymbol{y}|^{3}} \mathrm{~d} \Sigma_{x}$

where $\boldsymbol{n}^{f}$ is still the outwards normal to the fluid and $\alpha_{f}$ is either equal to $4 \pi$ if the point $\boldsymbol{y} \notin \partial \Omega$, or equal to the solid angle of the fluid portion delimited by the boundary $\partial \Omega$ at point $\boldsymbol{y}$ if $\boldsymbol{y} \in \partial \Omega$. Let us remark that, if domain $\Omega$ extends to infinity, but the function $\varphi$ satisfies Sommerfeld's conditions, this expression is still correct. As $\psi_{1}$, defined by (15), has such properties, using this relation provides the following integral equation $^{5}, \forall \boldsymbol{y} \in \Sigma$ :

$\alpha_{f(\boldsymbol{y})} \psi_{1(\boldsymbol{y})}-\int_{\Sigma} \psi_{1(\boldsymbol{x})} \frac{(\boldsymbol{x}-\boldsymbol{y}) \cdot \boldsymbol{n}_{x}^{f}}{|\boldsymbol{x}-\boldsymbol{y}|^{3}} \mathrm{~d} \Sigma_{x}=\int_{\Sigma} \frac{\dot{\boldsymbol{\xi}}^{\prime} \cdot \boldsymbol{n}_{x}^{f}}{|\boldsymbol{x}-\boldsymbol{y}|} \mathrm{d} \Sigma_{x}$

The BEM allows discretizing this equation by using interpolation functions defined on a mesh of the interface $\Sigma$. By collocating the points $\boldsymbol{x}$ and $\boldsymbol{y}$ at the nodes of this mesh, we can obtain a system of equations written under the following matrix form:

$([\alpha]-s[H])\left\{\psi_{1}\right\}=s\left[G_{n}\right]\left\{\dot{\boldsymbol{\xi}}^{\prime}\right\}$

where $\left\{\boldsymbol{\xi}^{\prime}\right\}$ and $\left\{\psi_{1}\right\}$ are vectors containing the values of fields $\boldsymbol{\xi}^{\prime}$ and $\psi_{1}$ at the mesh nodes, $[\alpha]$ is a diagonal matrix containing the values of $\alpha_{f}$ at these same nodes, $s=\boldsymbol{n}^{f} \cdot \boldsymbol{n}^{E F}$ is equal to \pm 1 depending on the sens of the normal $\boldsymbol{n}^{E F}$, defined by the mesh elements, with respect to the fluid outwards normal $\boldsymbol{n}^{f}$. Matrices $[H]$ and $\left[G_{n}\right]$ are defined by

$\forall \boldsymbol{y}_{i} \in \Sigma: \int_{\Sigma} \psi_{1}(x) \frac{\left(\boldsymbol{x}-\boldsymbol{y}_{i}\right) \cdot \boldsymbol{n}_{x}^{E F}}{\left|\boldsymbol{x}-\boldsymbol{y}_{i}\right|^{3}} \mathrm{~d} \Sigma_{x} \simeq \sum_{j=1}^{N_{\Sigma}} H_{i j} \psi_{1 j}$

$\forall \boldsymbol{y}_{i} \in \Sigma: \int_{\Sigma} \frac{\dot{\boldsymbol{\xi}}_{x}^{\prime} \cdot \boldsymbol{n}_{x}^{E F}}{\left\|\boldsymbol{x}-\boldsymbol{y}_{i}\right\|} \mathrm{d} \Sigma_{x} \simeq \sum_{j=1}^{N_{\Sigma}} G_{n i j} \dot{\boldsymbol{\xi}}_{j}^{\prime}$

\footnotetext{
${ }^{5}$ As every unknown or operator is now defined on the initial geometry $\Sigma_{0}$, the subscript ${ }_{0}$ will be droped in the following.
}

If we consider an external fluid, the unicity of $\psi_{1}$ is ensured by the far-field condition and system (27) can be inverted, but in the case of an internal fluid, the unicity condition $\ell\left(\psi_{1}\right)=0$ must be specified and taken into account to make the system invertible. We can choose to impose that $\psi_{1}\left(\boldsymbol{x}_{1}\right)=0$ on a specific node $\boldsymbol{x}_{1}$. Then, after discarding the row and column associated with this degree of freedom (obtained matrices will be denoted with a subscript ${ }_{2}$ ), system (27) becomes invertible and yields

ext. fluid: $\left\{\psi_{1}\right\}=\left(s^{e}\left[\alpha^{e}\right]-[H]\right)^{-1}\left[G_{n}\right]\left\{\dot{\boldsymbol{\xi}}^{\prime}\right\}$

int. fluid: $\left\{\psi_{1}\right\}=\left[\begin{array}{c}0 \cdots \cdots \cdots \cdots \cdots \cdots \\ \left(s^{i}\left[\alpha^{i}\right]_{22}-[H]_{22}\right)^{-1}\left[G_{n}\right]_{2}\end{array}\right]\left\{\dot{\boldsymbol{\xi}}^{\prime}\right\}$

where $s^{e}$ and $\alpha^{e}$ (respectively $s^{i}$ and $\alpha^{i}$ ) are the values of $s$ and $\alpha$ obtained for an external fluid (respectively for an internal fluid). Let us remark that $s^{e}=-s^{i}$ and $\alpha^{e}=4 \pi-\alpha^{i}$. Furthermore, the fact that (27) has, for an internal fluid, an infinity of solutions up to an additive constant, implies that

$\left(\left[\alpha^{i}\right]-s^{i}[H]\right)\{1\}=\{0\} \Rightarrow\left\{\alpha^{i}\right\}=s^{i}[H]\{1\}$

which yields a simple way to compute the interior solid angles $\alpha^{i}$.

By introducing the matricial expression of $\left\{\psi_{1}\right\}$ (29) in the classical FEM discretization of the fluidstructure coupling operator $C(\psi, \delta \boldsymbol{\xi})$ defined by

$C(\psi, \delta \boldsymbol{\xi})=\int_{\Sigma} \psi \boldsymbol{n}_{0}^{f} . \delta \boldsymbol{\xi} \mathrm{d} \Sigma_{0} \simeq{ }^{\mathrm{t}}\{\delta \boldsymbol{\xi}\}[C]\{\psi\}$

we can finally construct the matrices associated with the internal and external fluid added mass operators defined by (26):

ext. fluid: $\left[M_{F}^{e}\right]=-\rho[C]\left(s^{i}\left[4 \pi-\alpha^{i}\right]+[H]\right)^{-1}\left[G_{n}\right]$

int. fluid: $\left[M_{F}^{i}\right]=\rho[C]_{2}\left(s^{i}\left[\alpha^{i}\right]_{22}-[H]_{22}\right)^{-1}\left[G_{n}\right]_{2}$

Let us remark that, contrary to FEM, BEM provides non symmetric expressions for the fluid added mass matrix (and a numerical symmetrization step is then recommended).

An axisymmetric ellipsoid geometry is used as a validation test case (see figure 2). Although the presented approach allows constructing the added mass operator associated with any deformation of the structure, analytical values of the added mass and inertia associated with fluid outside and inside ellipsoids only exist for rigid translations and rotations, that is why, we will only consider this kind of motions here. The results are summarized in table 1 and show a very good agreement with the reference values calculated from the analytical formulae given by (Brennen 2006). 


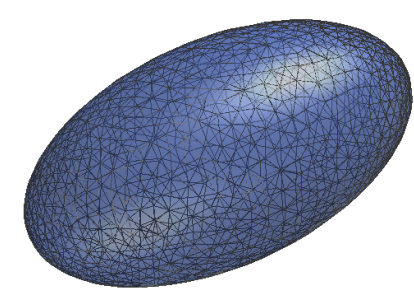

Figure 2: Ellipsoid mesh used for a test case $(a=2 b=2 . m)$

\begin{tabular}{|c|c|c|}
\hline & int. fluid & ext. fluid \\
\hline$m_{\text {longi. }}$ & $8320 .(0.7 \%)$ & $1739 .(1.1 \%)$ \\
\hline$m_{\text {transv. }}$ & $8319 .(0.7 \%)$ & $5826 .(1.2 \%)$ \\
\hline$I_{\text {pitch/yaw }}$ & $2987 .(0.9 \%)$ & $1971 .(1.7 \%)$ \\
\hline$I_{\text {roll }}$ & $\sim 0$. & $\sim 0$. \\
\hline
\end{tabular}

Table 1: Mass and inertia added by an internal or external fluid (water) on an ellipsoid, computed by BEM (error with respect to analytical values in \%)

\section{EXPERIMENTAL SETUP}

To explore the validity domain of the potential flow assumption for the fluid perturbations, on which our added mass model is established, an experimental setup has been realized (see figure 3). An articulated rod mechanism allows transforming the rotation of a motor into a yaw oscillation of an ellipsoid mockup, immerged in water. From the measurements provided by a torque meter and an accelerometer, the apparent inertia of the ellipsoid is obtained and after subtracting the corresponding solid inertia, the external fluid added inertia is deduced (no internal fluid here) and compared with the computed value: the ratio between them, denoted as $C_{m}$ is represented on figure 4 . By changing the length ratio between the rods of the mechanism, it is possible to observe the dependency of the results on the amplitude of the oscillation (evolution in $\mathcal{D}$ ) and to test the limits of the small pertubation assumption. Furthermore, by decreasing the motor rotation speed, we can reduce the value of the Stokes number $S t$ and observe the influence of the viscosity on the added mass prediction to determine the validity domain of the potential fluid model. Unfortunately, the present setup did not allow us to explore a wide range of amplitudes and our results seem not to be very sensitive to parameter $\mathcal{D}$. On the other hand, figure 4 shows a clear influence of the Stokes number on the fluid added mass. It provides the interesting result that for $S t>3 . \times 10^{4}$, the perfect fluid model gives quite good results, whereas its quality decreases rapidly under this value. This limit seems acceptable for the target application, i.e. aerostats in flight, since, for such systems the classical Stokes number is about $1 . \times 10^{7}$, which tends to validate this approach currently used in aerostat conception.

\section{CONCLUSIONS}

In this paper, the linearized equations of a structure vibrating in contact with a low-viscosity fluid (in per-

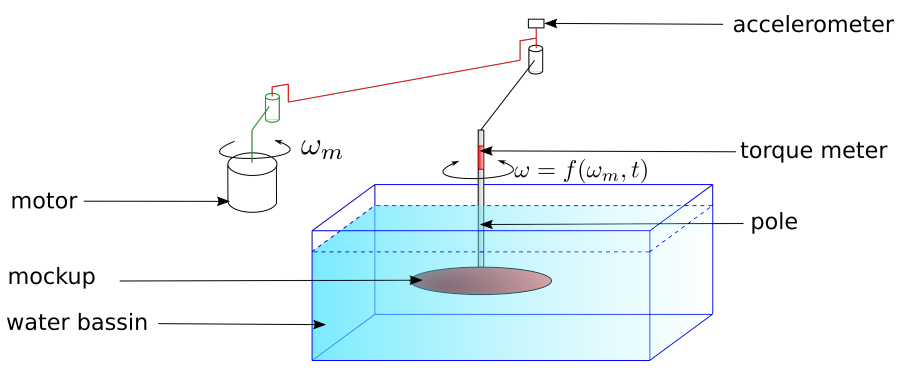

Figure 3: Diagram of experimental setup

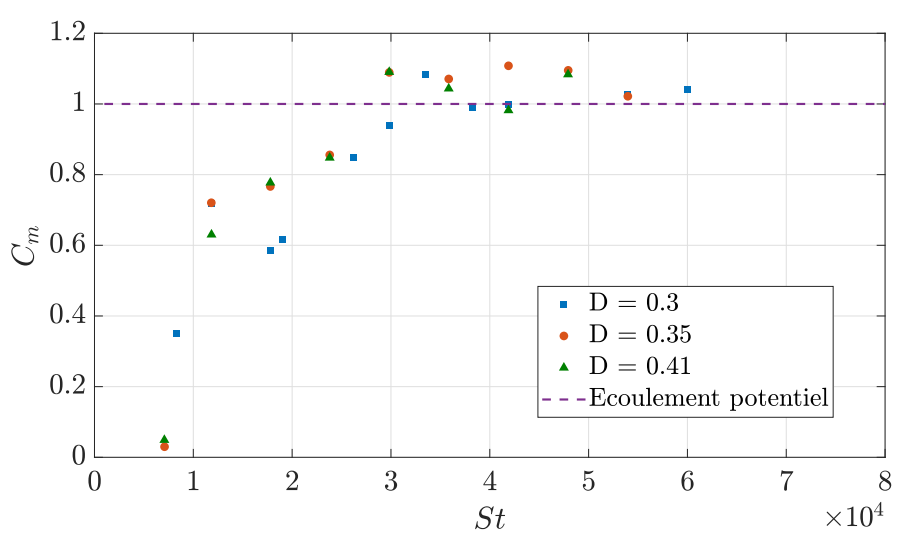

Figure 4: Influence of Stokes $(S t)$ and Displacement $(\mathcal{D})$ numbers on fluid added inertia $\left(C_{m}\right.$ is the ratio between the measured and computed values)

manent flow) have been reminded and the fluid added mass, stiffness and damping operators have been exhibited, with a particular attention about the assumptions needed to justify the potential model we used for the flow perturbations (especially in terms of displacement and Stokes numbers). A focus has been made on the fluid added mass operator, which, in this context, does not depend on the fluid permanent flow velocity field. A BEM approach has been proposed to numerically compute the added mass operators associated with fluids outside or inside deformable structures. An axisymmetric ellipsoid geometry has been used for numerical validations but also for experimental measurements dedicated to the characterization of the validity domain of our approach.

\section{REFERENCES}

Barenblatt, G. I. (2014). Flow, Deformation and Fracture: Lectures on Fluid Mechanics and the Mechanics of Deformable Solids for Mathematicians and Physicists. Cambridge Texts in Applied Mathematics.

Brennen, C. E. (2006). Internet book on fluid dynamics. California Institute of Technology. http://brennen.caltech.edu/fluidbook/.

Hackbusch, W. (1995). Integral Equations - Theory and Numerical Treatment. Birkhauser.

Hembree, B. \& N. Slegers (2011). Tethered aerostat modeling using an efficient recursive rigi-body dynamics approach. $J$. of Aircraft 48(2), 623-632.

Morand, H. J.-P. \& R. Ohayon (1995). Fluid-Structure Interaction: Applied Numerical Methods. Chichester: Wiley.

Schotté, J.-S. \& R. Ohayon (2009). Various modelling levels to represent internal liquid behaviour in the vibratory analysis of complex structures. CMAME 198, 1913-1925. 